\title{
DIRECT NUMERICAL SIMULATION OF WAKES WITH VIRTUAL CYLINDERS
}

\author{
Jorge H. Silvestrini ${ }^{1}$ \\ Departamento de Engenharia Mecânica e Mecatrônica, \\ Faculdade de Engenharia, \\ Pontifífica Universidade Católica do Rio Grande do Sul \\ Av. Ipiranga 6681, 90619-900 Porto Alegre - RS, Brasil \\ Eric Lamballais ${ }^{2}$ \\ Laboratoire d'Etudes Aérodynamiques UMR 6609, \\ Université de Poitiers, \\ Téléport 2 - Bd. Marie et Pierre Curie B.P. 30179 \\ 86962 Futuroscope Chasseneuil Cedex, France
}

\begin{abstract}
We use the virtual boundary technique in a context of Direct Numerical Simulation of turbulent flows around cylinders. This approach allows the imposition of a no-slip boundary condition within the flow field by a feed-back unsteady forcing term added to the momentum equation. In this paper, we consider the flow around a single cylinder with upstream conditions corresponding to a constant velocity flow or a mixing layer flow. Preliminary results show the capabilities of the virtual boundary technique to take complex obstacles into account realistically by the use of a simple Cartesian grid along with accurate finite difference method.
\end{abstract}

\section{INTRODUCTION}

For Direct Numerical Simulation (DNS) or Large Eddy Simulation (LES) applications, the best numerical strategy to consider complex geometry flows remains to be determined. Schematically, the main difficulty is to obtain an accurate description of the turbulent structure dynamics with a realistic shape of the body geometry. In engineering flow simulations, the description of the external geometry is traditionally favoured by the use of body-fitted curvilinear or unstructured grids. The major drawbacks of such approach are the considerable increase of the computational cost and the significant degradation of the accuracy. On the other hand, if regular grids are used, efficient code can be used (in terms of computational cost and accuracy) but is a priori limited to simple geometry. This paper presents results obtained from an alternative approach which consist in using accurate and efficient code to perform simulations of complex geometry flow on a regular grid. Such a possibility is offered by the immersed-boundary technique (see reference [1] for recent developments) which is treated in this work by the introduction of a body-force field in the momentum equation.

\footnotetext{
${ }^{1}$ jorgehs@em.pucrs.br

${ }^{2}$ lamballais@univ-poitiers.fr
} 


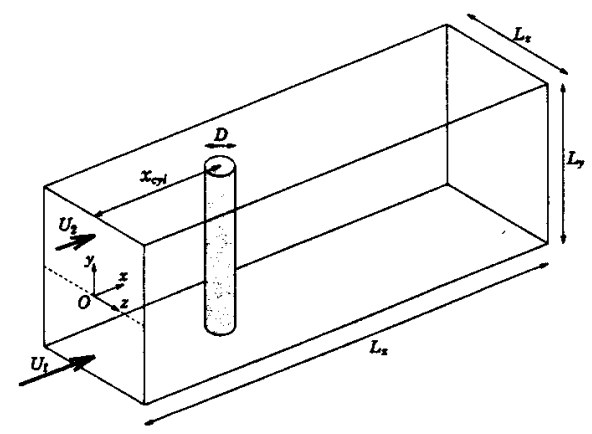

Figure 1: Schematic view of the flow configuration

\section{FLOW CONFIGURATION AND PARAMETERS}

Flows around a circular cylinder of diameter $D$ are considered in a Cartesian frame of reference $\mathcal{R}=(0 ; x, y, z)$. The cylinder axis is oriented along the vertical direction $y$ at the intersection between the streamwise section $x_{\text {cyl }}$ and the spanwise one $z=0$ (see figure 1). At the inflow section, a plane mixing layer flow between two streams of velocities $U_{1}$ and $U_{2}$ is imposed, the associated mean shear being aligned with the $y$-direction. The mean velocity profile $U(y)$ at $x=0$ is given by an hyperbolic tangent with

$$
U(y)=\frac{U_{1}+U_{2}}{2}-\frac{U_{1}-U_{2}}{2} \tanh \left(\frac{2 y}{\delta_{\omega_{i}}}\right)
$$

where $\delta_{\omega_{i}}$ is the inflow vorticity thickness. The difference and convective velocities are respectively noted $\Delta U=U_{1}-U_{2}$ and $U_{c}=\left(U_{1}+U_{2}\right) / 2$. The three fundamental nondimensional parameters of the present problem are the ratio of velocities $\lambda=\Delta U / 2 U_{c}$, the Reynolds number $R e=U_{\mathrm{c}} D / \nu$ and the ratio of lengths $H=D / \delta_{\omega_{i}}$. At the downstream of the cylinder, the present flow will consist of an interaction between a mixing layer and a wake of orthogonal mean shear directions, that is, a truly three-dimensional flow (without any homogeneous direction). Note that small values for $\lambda$ correspond to tlow regimes where mixing layer effects are moderate, while the case $\lambda=0$ denotes the case where a simple uniform flow, of constant velocity $U_{c}$, is imposed at the inflow section. In the present paper, two turbulent flows around the same cylindrical geometry are considered. The first simulation (UWAKE) corresponds to a wake formed by a uniform constant flow as inflow condition and the second (MLWAKE) is a turbulent wake forced by a mixing layer flow. The flow configurations for the two simulations are summarized in Table 1.

\section{NUMERICAL METHODOLOGY}

Governing equations

We consider the incompressible Navier-Stokes equations

$$
\begin{aligned}
\frac{\partial \mathbf{u}}{\partial t} & =-\frac{1}{\rho} \nabla p-\omega \times \mathbf{u}+\nu \Delta \mathbf{u}+\mathbf{f} \\
\nabla \cdot \mathbf{u} & =0
\end{aligned}
$$

where $\nu$ is the kinematic viscosity, $p(\mathbf{x}, t)$ the modified pressure field, $\mathbf{u}(\mathbf{x}, t)$ and $\omega(\mathbf{x}, t)$ being respectively the velocity and vorticity fields. The external force field $\mathbf{f}(\mathbf{x}, t)$ is 


\begin{tabular}{|c|c|c|c|c|c|c|c|}
\hline Test Run & $R e$ & $\left(L_{x}, L_{y}, L_{z}\right)$ & $x_{c y l}$ & $\lambda$ & $H$ & $\left(n_{x}, n_{y}, n_{z}\right)$ & $(\Delta x, \Delta y, \Delta z)$ \\
\hline UWAKE & 200 & $(16 \mathrm{D}, 4 \mathrm{D}, 8 \mathrm{D})$ & $2.5 \mathrm{D}$ & 0 & 0 & $(193,48,96)$ & $(\mathrm{D} / 12, \mathrm{D} / 12, \mathrm{D} / 12)$ \\
MLWAKE & 300 & $(28 \mathrm{D}, 8 \mathrm{D}, 16 \mathrm{D})$ & $13 \mathrm{D}$ & 0.5 & 1 & $(505,144,193)$ & $(\mathrm{D} / 18, \mathrm{D} / 12, \mathrm{D} / 18)$ \\
\hline
\end{tabular}

Table 1: Flow configurations and simulation parameters

used here to generate the "virtual" cylinder, its form will be given below. The previous equations are directly solved, in the context of the DNS, on a non-staggered grid with the number of grid points and mesh spacing shown in Table 1.

\section{Description of the code}

Sixth-order compact centered difference schemes are used [4] to evaluate all spatial derivatives, except near the in- and outflow boundaries where single sided schemes are employed for $x$-derivative calculation. Time integration is performed with a third-order low-storage Runge-Kutta method [5]. The incompressibility condition (3) is ensured with a fractional step method via the resolution of a Poisson equation for the pressure. For the UWAKE run, periodicity is imposed in the two transverse directions. For the MLWAKE run, freeslip boundary conditions are applied to $y= \pm L_{y} / 2$ using symmetry and anti-symmetry considerations while periodicity is imposed in the $z$-direction. This set of lateral boundary conditions presents two advantages: (i) first, it allows the preservation of the global accuracy of the $y$ and $z$-derivatives calculation by the use of the same numerical scheme in the whole computational domain; (ii) secondly, it offers the possibility to verify (in the discrete sens) at each sub-time step the incompressibility condition (3) up to machine accuracy with the aid of a Poisson solver based on a direct and partially spectral method. The outflow boundary conditions $\left(x=L_{x}\right)$ is deduced by solving a simplified convection equation

$$
\frac{\partial \mathbf{u}}{\partial t}+U_{b} \frac{\partial \mathbf{u}}{\partial x}=0
$$

where $U_{b}$ is an approximate convection velocity of the main structures in the outflow region, $U_{b}$ being the same at each node of the exit plane. For inlet conditions, small random perturbations with a given kinetic energy spectrum are superimposed on the inflow mean velocity profile to mimic residual turbulence upstream to the cylinder.

\section{Virtual boundary approach}

In this study, we use a virtual boundary technique originally proposed by Goldstein et al. [2]. This approach allows the imposition of no-slip boundary condition within the flow field by a feed-back unsteady forcing term $\mathbf{f}(\mathbf{x}, t)$ added to the momentum equation (see equation 2). These forces are chosen to lie along a desired surface and to have a magnitude and direction opposed to the local flow. Here, we use a forcing term acting on location $x_{3}$ corresponding to the presence of a virtual body at the same position, with

$$
\mathbf{f}\left(\mathbf{x}_{\mathbf{s}}, t\right)=\alpha \int_{0}^{t} \mathbf{u}\left(\mathbf{x}_{\mathbf{s}}, t^{\prime}\right) d t^{\prime}+\beta \mathbf{u}\left(\mathbf{x}_{\mathbf{s}}, t\right)
$$

where $t$ is the time and $(\alpha, \beta)$ are negative constants having dimensions of $1 / T^{2}$ and $1 / T$, respectively. Such a formulation allows to enforce $\mathbf{u}=0$ on the immersed boundary if $(|\alpha|,|\beta|)$ are large enough. In this work, we have used the largest possible values for $(|\alpha|,|\beta|)$ without introducing additional constraint in the stability limit for the time step which remains only constrained by CFL criterion (see reference [2] for more details). For 


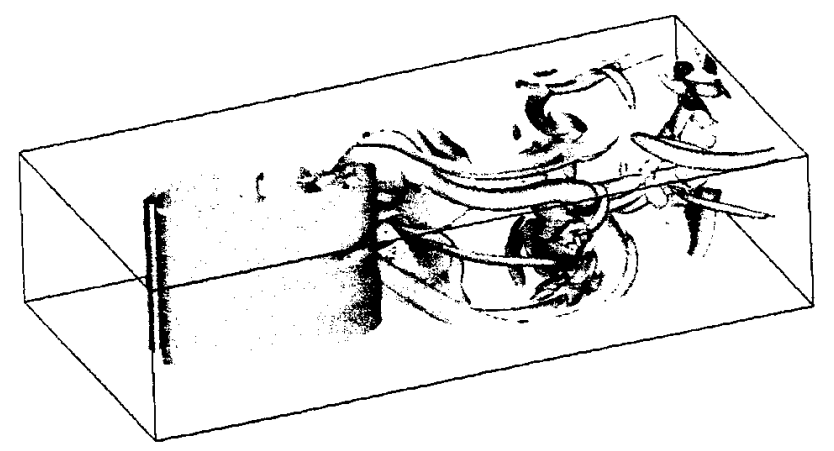

Figure 2: Perspective view showing isosurfaces of the vorticity modulus - Test run UWAKE

present numerical methods, the use of accurate centered schemes for spatial discretization (without numerical dissipation) impose a particular care in the treatment of forcing. Hence, if $\mathbf{f}(\mathbf{x}, t)$ is not smooth enough in space, significant spatial oscillations are produced. To eliminate this difficulty, we follow the procedure proposed by Goldstein $e t$ al. [2] (in a context of spectral methods) by the use of a spatial smoothing and filtering. The spatial smoothing is applied on $\mathbf{f}(\mathbf{x}, t)$ by the imposition of vanishing force in the adjacent points of the body surface with a Gaussian distribution. The spatial filtering is performed at each sub-time step on the non-linear and forcing term of equation (2) using compact filters in the three directions of space. Filter coefficients are chosen to preserve the global accuracy of spatial discretization (sixth order) and to affect only the smallest scales of the flow [4]. Note that a secondary benefit of the filtering is that aliasing errors are reduced everywhere in the computational domain.

\section{RESULTS}

\section{Wake due to an uniform flow - Test run UWAKE}

Fig. 2 shows a perspective view of the vorticity modulus denoting the structural organization of the flow. The picture was taken at the end of the simulation, $t=32 \mathrm{D} / U_{c}$, corresponding to twice the time necessary for a structure to travel the longitudinal length $L_{x}$ of the computational domain with a velocity $U_{c}$. Despite the limited (marginal) resolution (see Table 1), the virtual boundary technique, along with accurate numerical methods, seems to reproduce qualitatively all the keys structures of a turbulent wake, like the periodic detachment of Karman vortices and the stretching of streamwise vortices or hairpins in-between.

\section{Wake due to a mixing layer flow - Test run MLWAKE}

This complex flow was study experimentally by Heitz [3]. Figures 3 and 4 show top and side views of the vorticity modulus at the time $t=68 D / U_{c}$. Fig. 3 shows, from left to right, the roll-up and pairing of Kelvin-Helmholtz structures of the mixing layer before the cylinder and the development of a wake behind it. Fig. 4 shows a side view of the same instantaneous flow. In front of the cylinder, the mixing layer present a significant curvature associating with an upward pressure gradient. Such a behaviour is in agreement with experimental observations of [3]. Behind the cylinder, the inverse tendency (downward pressure gradient) observed experimentally [3] is recovered in present simulation. The analysis of the vortical structures at successive instants reveals the presence of 


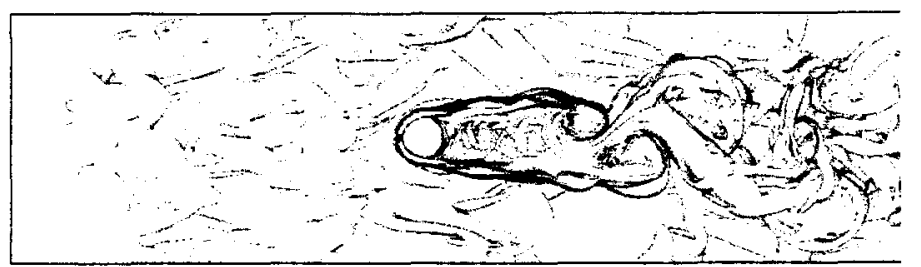

Figure 3: Top view showing isosurfaces of the vorticity modulus - Test run MLWAKE

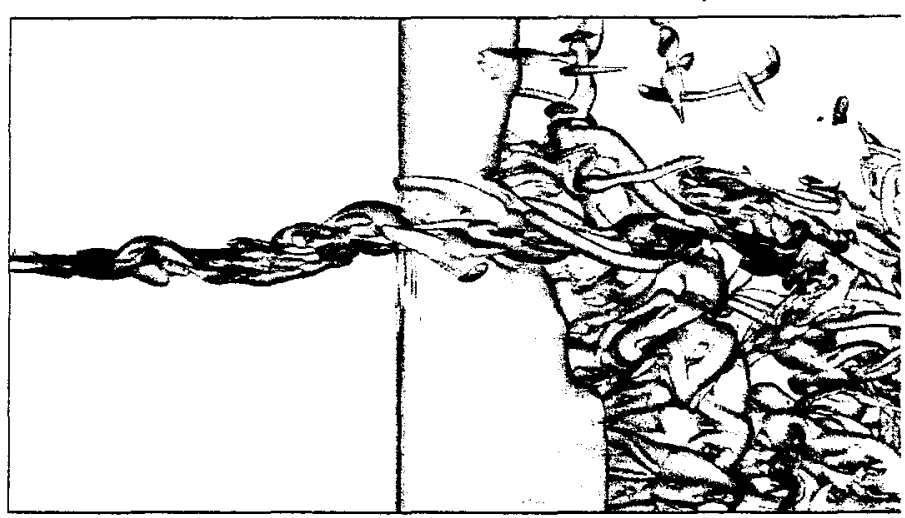

Figure 4: Side view showing isosurfaces of the vorticity modulus - Test run MLWAKE

oblique detachment of Karman vortices from the cylinder in an angle increasing during the downstream development of the flow. This behaviour seems to be due to the velocity difference $\Delta U$ between the two streams of the mixing layer which tend to transport each part of Karman vortices with their own convection velocity scaled by $U_{1}$ or $U_{2}$. From this interpretation, it can be anticipated that the velocity ratio $\lambda$ will be the main parameter to determine the inclination angle of the Karman vortices. Note that the presence of Karman structures crossing the mixing layer shows the existence of synchronization mechanisms between the slow and fast wakes, may be through viscous reconnection in the core of mixing layer. Finally, the complex nature of this flow as well as its considerable number of degrees of freedom is illustrated by a perspective view in Fig. 5 .

\section{CONCLUSIONS}

In this work, we have shown that an accurate and efficient numerical code allows to perform DNS of turbulent flows in presence of obstacles with a geometry which is not a priori well designed for the simple Cartesian grid used here. Such a result is possible by the use of virtual boundary technique introducing a new term in the equation to generate a no-slip boundary condition at the surface of the virtual body. Present results show that a realistic turbulent flow dynamic can be obtained in the presence of a single cylinder, if the treatment of the forcing term is done carefully. The interaction between a mixing layer and a wake (generated by the cylinder) is considered and preliminary results show that the mean structural features of this complex flow observed experimentally 


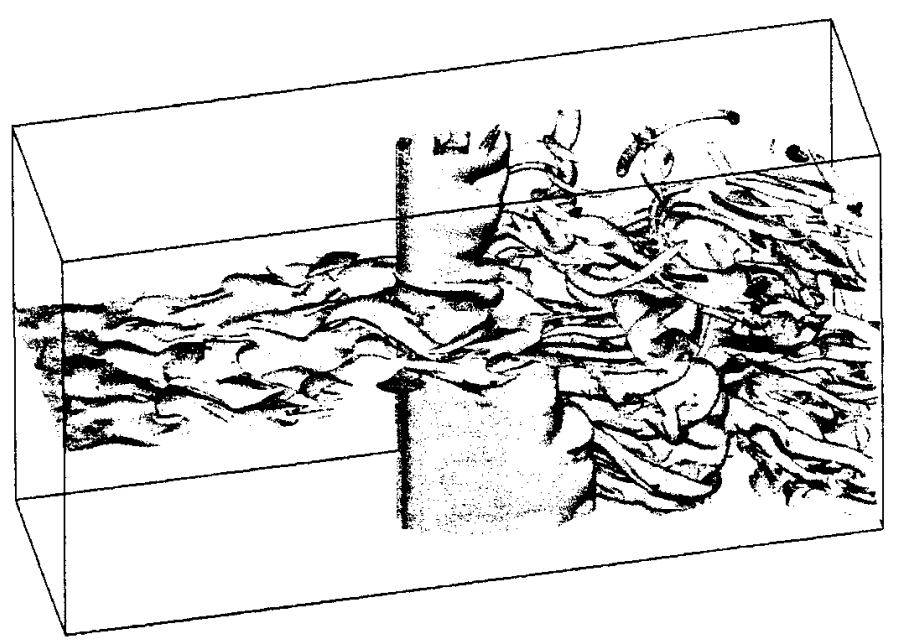

Figure 5: Perspective view showing isosurfaces of the vorticity modulus - Test run MLWAKE

are recovered numerically. This is the first time, in our knowledge, that such a threedimensional complex flow is reproduced by DNS. Now, the present simulation has to be continued in order to perform a statistical treatment of the fluctuating flow for quantitative comparisons with experiments. If a good quantitative agreement is found, it is clear that the potential of the present technique would be considerable, in terms of efficiency and simplicity, allowing to consider complex geometry (of industrial interest) without any loss of accuracy in the turbulent structure dynamics.

\section{REFERENCES}

[1] Fadlun, E. A., Verzico, R., Orlandi, P., and Mohd-Yusof, J. Combined immersed-boundary finite-difference methods for three-dimensional complex flow simulations, J. Comp. Phys., Vol. 161, 2000, págs. 35-60.

[2] Goldstein, D., Handler, R., and Sirovich, L. Modeling a no-slip boundary condition with an external force field, J. Comp. Phys., Vol. 105, 1993, págs. 354-366.

[3] Heitz, D. Etude expérimentale du sillage d'un barreau cylindrique se développant dans une couche de mélange plane turbulente. Thèse de l'Université de Poitiers, 1999.

[4] Lele, S. K. Compact finite difference schemes with spectral-like resolution, J. Comp. Phys., Vol. 103, 1992, págs. 16-42.

[5] Williamson, J. H. Low-storage Runge-Kutta schemes, J. Comp. Phys., Vol. 35, 1980 , págs. 48-56.

Acknowledgments This work was partially supported by the CEMAGREF, Rennes. One of the simulation, run MLWAKE, was performed at the IDRIS Computational Center at Orsay. 